\title{
FROM THE DESK OF EDITOR in CHIEF
}

(J Bangladesh Coll Phys Surg 2017; 35: 47)

Dear Fellows,

Seasons Greetings and Happy New Year.

I take he opportunity to thank all my Authors, Reviewers, Editors and Office Bearers for their fabulous support throughout 2016. You have made the last year a very successful year for JBCPS. We have been able to publish numerous wonderful articles that has been well appreciated hoe and abroad. New we look forward to take our beloved journal to a higher level in 2017.

Your all out support is my strength.

Thank you

Prof. Khan Abul Kalam Azad

Editor-in-Chief

Journal of BCPS 